\title{
Análisis de eficiencia en los procesos de las fábricas artesanales de muebles de madera
}

\section{Analysis of efficiency in the processes of artisanal factories of wooden furniture}

Julio Cesar Méndez Bravo

Universidad de Guayaquil, Ecuador

Manuel Alberto Méndez Bravo

Universidad de Guayaquil, Ecuador

Autor por Correspondencia: julio.mendezb@ug.edu.ec, ing.com.manuelmendez@hotmail.com Fecha de recepción: 19 de Enero de 2017 - Fecha de aceptación: 20 de Marzo de 2017

\section{Resumen}

La presente investigación utiliza una metodología cualitativa, ya que emplea el método inductivo mediante el análisis de un caso en particular para plantear un modelo a negocios con similares características, analiza el caso particular de la Mueblería "Muebles Deco-Arte Hogar" basados en la aplicación general de la literatura empleada por diferentes autores en otras investigaciones, en el que se analizan sus procesos de producción con el sistema de control de calidad y se propone un nuevo proceso eficiente de optimización en el área de producción y su organización del recurso humano y demás recursos para la producción de muebles.

Palabras claves: calidad; mejora continua; normas iso; procesos

\begin{abstract}
This research uses a qualitative methodology, employing the inductive method by analyzing a particular case to raise a model businesses with similar characteristics analyzes the particular case of the "Furniture Deco-Art Home" Mueblería based on the general application literature used by other authors in other studies, in which production processes are analyzed with its system of quality control and a new efficient process optimization proposed in the area of production and organization of human resources and other resources for furniture production.
\end{abstract}

Key Words: quality; continuous improvement; iso standards; processes 


\section{Introducción}

Como nos indica Parsons (1960), "las organizaciones son unidades sociales (o agrupaciones humanas) construidas intencionalmente y reconstruidas para alcanzar objetivos específicos". Esto quiere decir que las organizaciones se reorganizan o se re estructuran según se vayan cumpliendo sus objetivos, ya que las organizaciones no se pueden alterar, más bien se adapta a los cambios y a las situaciones.

Administrar se refiere sobre todo a planear y organizar la estructura de órganos y cargos que componen la empresa, dirigir y controlar sus actividades. (Chiavenato, 2001) Lo que se busca en esta etapa de mejora de procesos es realizar las actividades de la empresa de la forma planeada y establecer lineamientos y estrategias para que cada quien se desempeñe eficientemente en sus funciones, asumiendo con responsabilidad su rol en la empresa.

Para que esta investigación tenga un sustento teórico en el marco de las aplicaciones de los conceptos y mejoras planteadas, se recabo información sobre teorías relacionadas con la estructura organizacional, de las cuales se buscó encontrar o seleccionar las practicas más recomendables o fáciles de aplicar a la empresa y que sus conceptos no sean confusos para el mejor entendimiento de las personas que forman la organización.

Según Chiavenato, (2001) el Enfoque relacionado con la estructura organizacional son tres:

- Teoría clásica de Fayol

- Teoría de la burocracia de Weber

- Teoría estructuralista.

\section{Teoría clásica de Fayol habla de 6 funciones básicas:}

1. Funciones técnicas, relacionadas con la producción de bienes y servicios.

2. Funciones comerciales, relacionadas con la compra, venta e intercambio.

3. Funciones financieras, relacionadas con la búsqueda y gerencia de capitales.

4. Funciones de seguridad, relacionadas con la protección y preservación de los bienes y las personas.

5. Funciones contables, relacionadas con inventarios, registros, balances, costos y estadísticas.

6. Funciones administrativas, relacionadas con la integración desde la cúpula de las otras cinco funciones. Las funciones administrativas coordinan y sincronizan las demás funciones de la empresa, manteniéndose siempre por encima de ellas.

Las funciones del administrador son:

- Prever

- Organizar

- Mandar

- Coordinar

- Controlar

Teoría de la burocracia, presenta 7 dimensiones: 
1. 'Las actividades de la organización se definen por escrito y la organización opera de acuerdo con un conjunto de leyes o reglas que se aplican a todos los casos individuales sin excepción.

2. División de trabajo: cada participante tiene un cargo o posición definido con esfera especifica de competencia, con deberes oficiales y atribuciones estrictamente especificadas y delimitadas.

3. Principio de jerarquía: la burocracia se fundamenta en una jerarquía bien definida de autoridad. Cada empleado está sometido a órdenes impersonales que guían sus acciones para asegurar la obediencia. Cada función inferior está bajo control y supervisión de la más alta, garantizando unidad de control; cada empleado solo tiene un jefe. De ahí la estructura piramidal de la burocracia.

4. Impersonalidad: el empleado ideal establece relaciones impersonales con otros ocupantes de cargos. La burocracia enfatiza en los cargos y no en las personas que los ocupan pues aquellas entran y salen de la organización, pero los cargos permanecen, para garantizar su continuidad y perpetuación.

5. Competencia técnica: la selección y elección de los empleados de los empleados se basa en la competencia técnica y las calificaciones profesionales de los candidatos, no en las preferencias de orden personal. De ahí que se utilicen pruebas y concursos para llenar las vacantes o conceder ascensos. La burocracia es eminentemente meritocrática. El sistema también prevé el desarrollo de la carrera y las promociones se realizan de acuerdo al mérito funcional, dependiendo siempre del juicio del superior.

6. Separación entre propiedad y administración: los recursos utilizados por la administración burocrática para ejecutar sus tareas no son propiedad de los burócratas. La administración está separada de la propiedad de los medios de producción, pues el dirigente no es necesariamente dueño de la organización o de sus medios de producción, sino un profesional especializado en la administración de su propio negocio, y la dimensión del capital en las sociedades anónimas.

7. Profesionalización del empleado: los empleados de la burocracia son profesionales pues son especialistas, gracias a la división del trabajo son asalariados de acuerdo con sus funciones o posiciones jerárquicas, sus cargos constituyen su principal actividad dentro de la organización, son nombrados por el superior inmediato, sus contratos se firman a tiempo indefinidos, siguen carrera en la organización y no son propietarios de los medios de producción de la organización."

\section{Teoría estructuralista}

Enfoque múltiple y amplio en el análisis de las organizaciones, que las concibe como complejos de estructuras formales e informales. La innovación y el cambio son fuente de conflictos en las empresas y que el conflicto es una importante señal de vitalidad en las organizaciones. La administración de conflictos se convierte en un elemento esencial y de múltiples aplicaciones.

De las tres teorías antes mencionadas (Teoría clásica de Fayol, Teoría de la burocracia de Weber y la Teoría estructuralista) se hará énfasis en la Las funciones de seguridad, contables y Administrativas de la teoría clásica de Fayol, ya que se relacionan más a la interrelación entre las 
personas que forman la organización, a la manera de llevar los controles mediante el uso de inventarios y registros además de establecer las funciones administrativas coordinadas y sincronizadas entre los diferentes niveles de jerarquías.

Las funciones que establece que debe de tener el administrador que son de Prever, Organizar, Mandar, Coordinar y Controlar, también serán adoptadas y explicadas de forma más detallada, ya que no solo serán enfocadas al administrador como tal, sino que serán enfocadas para cada persona de la organización, ya que se debe de recordar que cada persona de la organización es responsable de un trabajo específico y como tal tiene que administrar su tiempo, recursos entregados y coordinar trabajo con demás miembros de su grupo de trabajo.

De la teoría Burocrática se enfocará en la Formalización, ya que si las ordenes o actividades están por escrito no existirán malos entendidos y las situaciones que se presenten en la organización se podrán resolver de forma rápida y clara, acorde a lo establecido en leyes o reglamentos de la organización.

La División de trabajo en la Teoría de Fayol también se aplicará, solo definiendo las competencias de los cargos. El Principio de jerarquía dentro de esta teoría se lo aplicara pero no como una manera de establecer qué cargo es superior a otro cargo, sino para tener definidos los roles y quien tiene la última palabra para la toma de decisiones en algún problema específico.

De la Teoría estructuralista que posee un enfoque múltiple y amplio en el análisis de las organizaciones, donde se establece que una organización que se innova genera cambios y que personas que forman parte de la organización son renuentes al cambio lo que puede generar conflictos internos, pero lo cual indica a su vez que dichos cambios son señales de mejoras y de la vitalidad de la organización y como tal, la administración de conflictos se convierte en un elemento esencial y de múltiples aplicaciones.

\section{Modelo de gestión de calidad}

Denning, (2010) define a la estrategia empresarial como "la determinación de la posición futura de la empresa, en especial frente a sus productos y mercados, su rentabilidad, su tamaño, su grado de innovación y sus relaciones con sus ejecutivos, sus empleados y ciertas instituciones externas"

Además Denning, (2010) define lo que se desea lograr con la empresa u organización, y que una estrategia empresarial puede lograr todos los puntos indicados en su concepto, pero lo más importante es que en dicha definición, toma en cuenta al producto, mercado, personal e instituciones externas.

Lo que nos permite apreciar es que un administrador, en una estrategia empresarial debe de tomar todos los aspectos posibles para generar cambios positivos en la organización, donde se involucran todos los aspectos, los cuales tienen una interrelación que permite un gran éxito o fracaso. Y uno de esos factores es el talento humano con el que se va a trabajar o con el que se está trabajando, tomando en cuenta que el caso de estudio de esta investigación se lo realiza en 
un negocio familiar, donde familiares del o de los dueños del negocio forman parte de la toma de decisiones o de las actividades a realizar.

Se requiere de una cooptación en los puestos estructurales de la organización.

Cooptación: proceso de inserción de nuevos individuos para mantener el liderazgo o la estructura de decisión de la política de una empresa, y evitar amenazas o presiones a su estabilidad existencial. (Selznick, 1979)

Muchas PYMES no cuentan con instructivo de modelo de gestión de calidad de procedimientos, sobre todo cuando una empresa chica inicia, o incluso, cuando llevan tiempo laborando, una empresa que desee permanecer en el mercado actual y quiera tener un crecimiento sostenido debe estrictamente llevar uno. Ya que al momento en que la empresa crece entran en conflictos cuando hay reclutamiento y selección de personal ya que es más complicado encontrar a la gente adecuada en el puesto apropiado si no están específicamente establecidas las actividades, prioridades, responsabilidades, limitantes, entre otras de cada puesto y persona.

Un Instructivo de Modelo de Gestión de Calidad de Procedimientos brinda muchas ventajas a la empresa y a todo el personal que trabaja en ella, entre ellas:

- Permite medir la productividad de forma matemática.

- Nos facilita la contratación de la persona adecuada para el puesto adecuado

- Ahorra tiempo en capacitación

- Las actividades se desarrollan en base a tiempos medibles y se ahorran recursos materiales y humanos.

- Permite que los departamentos sepan cual es la tarea de cada cual, de esta forma el trabajo en conjunto fluye de una manera adecuada

- Se convierte en una empresa con orden y efectiva

- Es más sencillo para el dueño delegar responsabilidades al personal a cargo de cada taller así como de parte los maestros artesanos a los oficiales principales y de ellos a sus oficiales.

- Se mejora la calidad en las diferentes áreas, una de las que mayor requerimiento lleva es saber cómo brindar un buen servicio pre y post venta las que tendrán una buena calificación por parte del cliente y serán recomendados.

Como se observa es un conjunto grande de ventajas tener un instructivo de modelo de gestión de calidad de procedimientos que delinee todo de forma concisa y precisa.

Basado en los datos del caso de estudio y hechos se plantea la siguiente pregunta: “¿Cuán eficientes son las fábricas artesanales de muebles de madera en sus procesos productivos y operativos?"

Plantear procesos eficientes en la mejora de la calidad de los servicios y propender al mejoramiento de la imagen en la industria artesanal orientados a la fabricación de muebles de madera.

\section{Variables:}


- Variable Dependiente: Muebles de Madera

- Variable Independiente: Fabricación Artesanal

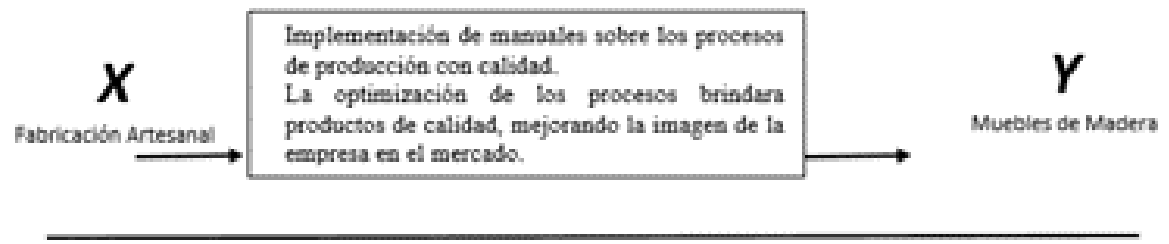

Figura1: Variables

Elaborado por: Autores

\section{Hipótesis}

Si se implementa procesos eficientes acorde a las necesidades de las micro empresas de fabricación artesanales de muebles de madera, mejorará la eficiencia en la calidad de los productos y servicios, mejorando la imagen de la empresa para con sus clientes, con lo que ampliará su base de clientes.

Se propone realizar un modelo de gestión de calidad que permita mejorar la eficiencia en los procesos operativos y administrativos de las mueblerías artesanales que den como resultado Fábricas artesanales eficientes en la elaboración de muebles de madera. Dado que la investigación se basa en un negocio familiar, con falta de orden administrativo y de calidad en sus procesos, las propuestas de mejoras van direccionadas a perfeccionar procesos para hacerlos más eficientes, mediante la implementación de pasos en ciertos procesos, la omisión de pasos innecesarios y con esto tener una mejor organización.

\section{Organigrama propuesto.}

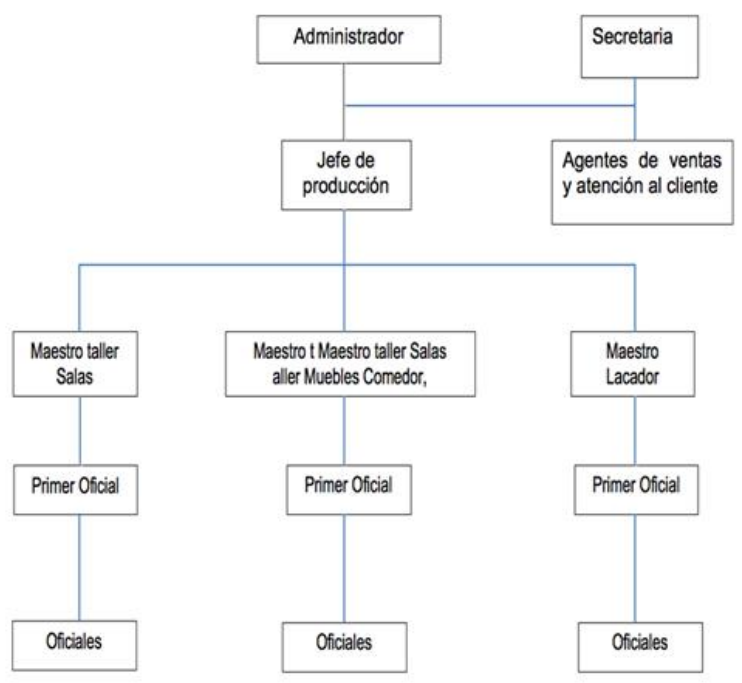

Figura 2: Organigrama de mueblería Elaboración: Autores

Creación de tablas de control, inventario, producción y de personal. 
Para poder llevar un mejor control de los recursos del negocio, es necesario un control ordenado, que permita al administrador y a los empleados saber con exactitud la cantidad de materia prima e insumos que tienen a disposición para trabajar, así como su avance en la producción y sobretodo monitorear el buen desempeño del personal.

"Talleres de trabajo.- Producción de pequeñas series de una gran cantidad de productos diferentes, la mayor parte de los cuales requieren una serie o secuencia distinta de pasos de procedimientos". (Chase, Aquilano, Jacobs, 2001)

\section{Tabla 1: Especificaciones y costos de la calidad}

\begin{tabular}{ll}
\hline Atributo & Característica \\
\hline Desempeño & Características de un producto primario o servicio. \\
\hline Características & Detalles adicionales, accesorios, características secundarias. \\
\hline Confiabilidad & Consistencia con el tiempo extra de desempeño, probabilidad de fallar. \\
\hline Durabilidad & Vida útil. \\
\hline Servicios & Fácil de reparar \\
\hline Respuesta & Características de la interrelación humana. (velocidad, educación, competencia) \\
\hline Estética & Características sensoriales. (Sonido, sensación, aspectos.) \\
\hline Reputación & Desempeño pasado y otros intangibles (calidad y percibida) \\
\hline
\end{tabular}

\section{Elaboración: El Autores}

\section{Procesos Propuestos:}

Diagrama de procedimientos De Almacenamiento de Madera e Insumos

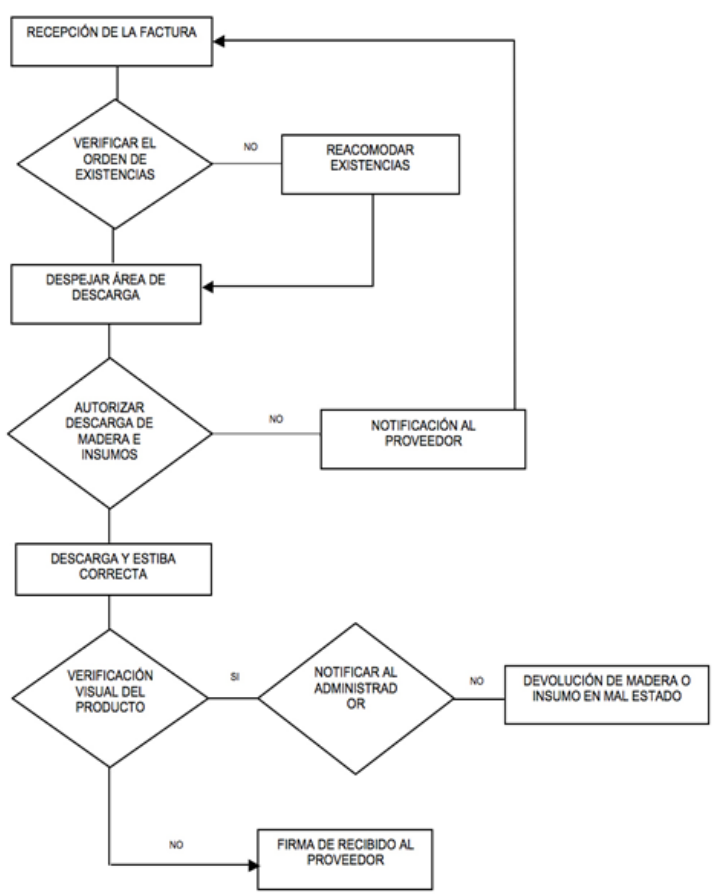

Diagrama de procedimientos de órdenes de producción Proceso General

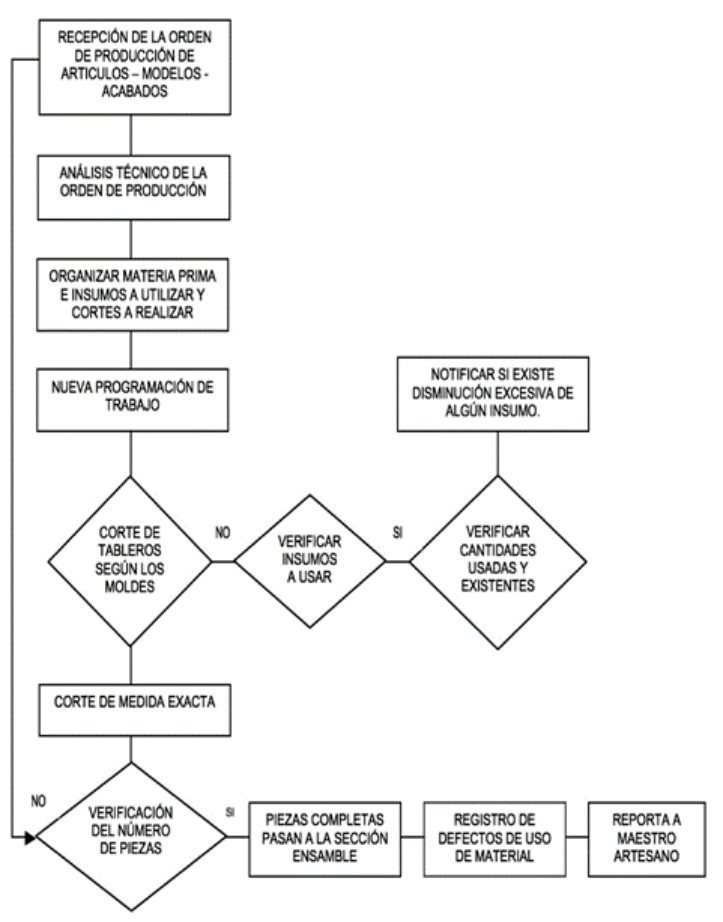


Figura 3: Diagrama de procedimientos de Almacenamiento Elaborado: Autores

Diagrama de procedimientos de lacados Proceso General

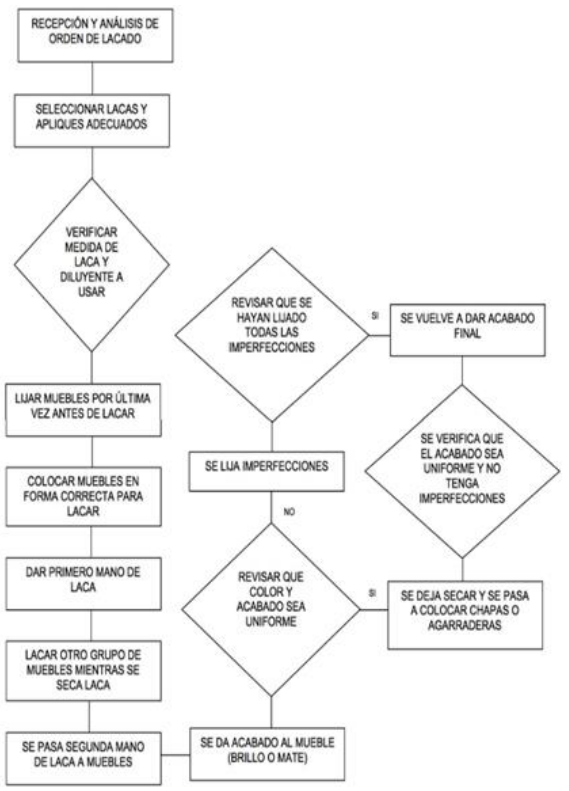

Figura 5: Diagrama de procedimientos de Lacados

Elaborado: Autores
Figura 4: Diagrama de procedimientos de Ordenes de Producción Elaborado: Autores

Diagrama de procedimientos de Armado y herraje

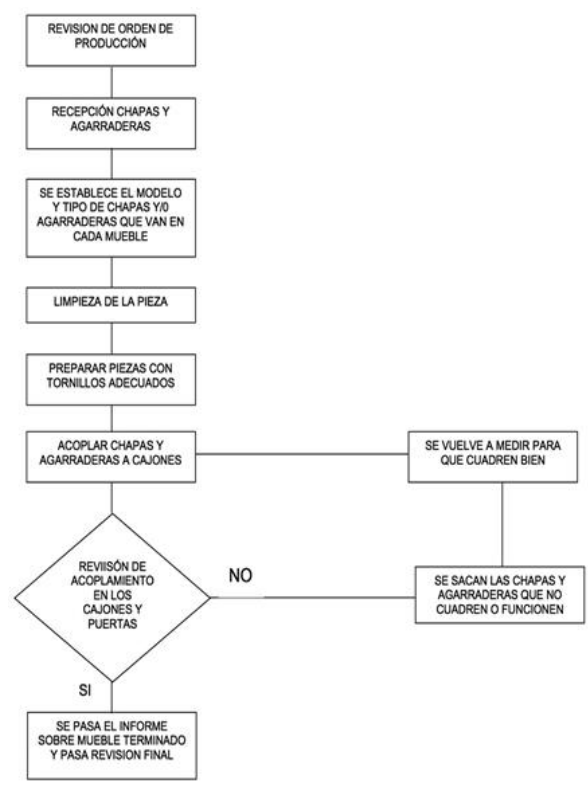

Figura 6: Diagrama de procedimientos de Armado y herraje Elaborado: Autores

\section{Talleres motivacionales y de capacitación}

Su más importante inversión es cualquier cosa que eduque a la gente y que ponga más en sus cabezas y le dé más motivación a sus corazones. (Hammer, 1996). El dictar talleres de motivación y capacitación surge de la necesidad de tener personal más capacitado, consciente y comprometido con las necesidades de la empresa.

Los talleres de capacitación no solo serán relacionados con su desarrollo laboral, este tipo de talleres tendrá un enfoque más relacionado a la preparación personal e interpersonal en la empresa y en sus hogares.

El incumplimiento en algunos trabajos se debe muchas veces a la falta de comunicación entre el personal de los diferentes talleres y el realizar actividades donde interactúan y comparten experiencias y se logra un ambiente mucho más ameno y afable y con esto una mejor comunicación y colaboración entre el personal de los diferentes talleres.

En lo referente a la capacitación y motivación con actividades relacionadas a su hogar es básicamente para que entiendan la importancia del amor y respeto para su hogar y sus familias, ya que al ser jefes de hogares es necesario que brinden buenos ejemplos en sus hogares, 
adicional a esto capacitarlos en la buena administración del presupuesto familiar, como organizar presupuestos semanales y como priorizar las necesidades.

El objetivo de estos talleres es básicamente tener personal más organizado y responsable para con la empresa, un empleado feliz y sin problemas en el trabajo y en su hogar, todo esto permitirá contar con un obrero más eficiente y eficaz al realizar sus labores, lo que se refleja en una mayor y mejor producción.

\section{Conclusiones}

El Modelo de Gestión de Calidad permite seguir delineamientos para tener una estructura sólida en su administración, desarrollo y trabajo de la empresa.En la actualidad las empresas o negocios que recién empiezan o que ya están en el mercado y se han mantenido en él, pero sin un crecimiento ordenado ni acorde a las necesidades del mercado actual, esto se debe a los administradores o las personas que dirigen el negocio no poseen la capacitación ni la preparación para ello, factor que puede determinar un decrecimiento en sus ventas y producción y la larga en el cierre de su negocio.

Con Modelo De Gestión de Calidad se espera que un negocio familiar rustico y desorganizado, se pueda transformar en una empresa ordenada con planes de crecimiento, expansión ordenada y estableciendo metas a corto y mediano plazo, preparándose para en lo posterior estar apto y capacitado para certificaciones de calidad en sus procesos de producción.

Un Modelo de Gestión de Calidad ofrece pasos y lineamientos específicos por área que se deben seguir para llegar a la organización y eficiencia de procesos. Así como el manejo que se debe de tener con el personal que labora en los procesos de fabricación y la mejora en la relación interpersonal en las áreas de trabajo. Una implementación correcta de un Modelo de Gestión de Calidad en el corto plazo eliminara la resistencia al cambio por parte del personal y esto permitirá a cualquier organización una mejor adaptación cuando la empresa desee certificarse.

El Modelo de Gestión de Calidad es un paso previo a la certificación ISSO, dado que se organiza por áreas, se disciplina al personal para trabajar de forma ordenada, armónica y eficiente, logrando concientizar a todo el personal desde los mandos altos a los medios, que un trabajo organizado y eficiente logra beneficios para la empresa lo que se refleja en beneficios para todo el personal. Se debe tener presente que un Modelo de Gestión de Calidad no solo trata de mejorar la calidad de los procesos sino que además de eso, también busca mejorar las relaciones interpersonales de todas las áreas de la empresa.

\section{Bibliografía}

Acuña Jorge (2004) - Mejoramiento de la Calidad: Un enfoque a los servicios, Editorial Tecnológica de Costa Rica, Costa Rica, Primera Edición,

CHASE Richard, AQUILANO Nicholas, Jacobs Robert (2001), Administración de Producción y Operaciones, Manufactura y servicios, Mc Graw- Hill, octava edición. 
Chiavenato Idalberto (1999), Recursos Humanos, Brasil, Edição Compacta, Quinta Edición

Chiavenato Idalberto (2001), Administración Procesos Administrativos, Brasil, Mc Graw Hill, Quinta Edición

Cortiñas (2008). Las siete herramientas para la calidad. Recuperado de http://www.apuntesgestion.com/productividad/concepto-productividad/

Crece Negocios (2011). Concepto de calidad. Recuperado de http://www.crecenegocios.com/concepto-de-calidad/

Denning Basil W. (2010), corporate planning: selected readings, United State, McGraw Hill, Onceava edición.

Fundación Ceprona (2011). Productividad. Recuperado de http://www.ceprona.org/organizacion/productividad.php

Gutierrez Cuauhtémoc (2004) - Administración Y Calidad, México, Editorial Ediciones Limusa S.A., Primera Edición

Hammer Michael (1996), Beyond Reengineering, United State, Crown Book

Izar Juan, González Jorge (2004) - Las Siete Herramientas Básicas De La Calidad, México, Universidad Autónoma De San Luis de Potosí, Primera Edición.

López (2009). El concepto de organización para nuestra pyme (I). Recuperado de http://www.pymesyautonomos.com/estrategia/el-concepto-de-organizacion-para-nuestrapyme-i

Mesa (2016). Fundamentos de sistema de gestión de calidad. Recuperado de http://www.euskosare.org/enpresak/kalitatea/definicion_conceptos_claves_sobre_calidad

Parsons Talcott (1960), Structure and Process in Modern Societies, United State, University of Michigan.

Selznick Philip (1979), “Cooptation” In Merlin B. Brinkerhoff Y Philip R. Kunz (Orgs.) Complex Organizations and Their Environments, Dubuque, Wm. C. Brown,

Senge Peter M. (2006), the Fifth Discipline: The Art and Practice of the Learning Organization, United State, and Currency Doubleday

Velasco (2010). Concepto de productividad. Recuperado de http://coyunturaeconomica.com/consejos/concepto-de-productividad

Zaid (2008). Conceptos de Productividad. Recuperado de http://www.letraslibres.com/mexico/conceptos-productividad. 